\title{
REASONABLE APPLICATION OF AUGMENTED REALITY IN ENGINEERING EDUCATION
}

\author{
J. Chlebusch, I. Köhler and C. Stechert ${ }^{\otimes}$ \\ Ostfalia University of Applied Sciences, Germany \\ $\bowtie$ c.stechert@ostfalia.de
}

\begin{abstract}
Augmented Reality (AR) is to be used extensively in today's digitized teaching in order to enable students to a more efficient learning. However, teaching content must not be only digitised, but must be communicated in a meaningful way. For this purpose, a generally valid flowchart has been developed, that allows lectures to choose the right content for AR experiences. In order to create this flowchart, empirical values from test runs were combined with pedagogically proven empirical values for good teaching.
\end{abstract}

Keywords: augmented reality (AR), case study, empirical studies

\section{Introduction and motivation}

Nowadays, Augmented Reality (AR) is applied at work in the industry, to support and improve production steps (Campbell et al., 2019; Davies, 2018). This is important to ensure that more complex products can be produced economically (Campbell et al., 2019; Thomas et al., 2018). For example, it is easier for production personnel to perform prescribed mountings of numerous wiring harnesses on airplanes, because their positions are displayed in the assembly by AR experiences (Davies, 2018). In engineering education it is important too, to make hidden information (e.g. inner forces, hidden parts of an assembly) visible to make it easier for the students to understand the connection between the real object and its theoretical abstraction (Slater, 2017; Probst and Ebner, 2018). Especially in the case of Mechanical Engineering, students must deal with understanding and developing complex products, even though most of them have only academical knowledge at this point of time. Therefrom, they could have problems to achieve the objectives in the practically oriented education in colleges of technology. Thus, a right application of AR could improve education by promoting efficient learning. Efficient learning does not only mean that the learning objectives are effectively achieved by the learning method, but also that a lower resource input (e.g. learning time) is required. Particularly in engineering education the lack of comprehension needs to be compensated in a short amount of time due to a fully packed curriculum in basic studies and the overall demand of a short duration of study. Therefore, in order to promote efficient learning, conventional teaching materials like lecture notes, projects, simulation games and so on can be supported more realistically by Augmented Reality applications (Thomas et al., 2018), after basic requirements have been elaborated (section 2).

A first experimental application of AR experiences was performed with students of Ostfalia university during the "machine elements" lecture in summer 2019 (Figure 1). It was intended as a feasibility study to gain knowledge about effort and usability. In this test run, 40 second year bachelor students 
were first introduced to three AR experiences using a smartphone app. The AR experiences showed the different gear variants "straight toothed" as well as "helical toothed" with and without profile shift. The basic structure of such gears, the differences between the mentioned gear types as well as the forces acting on the gears were explicitly visualised. The students had time to try out the experiences themselves and answer questions about handling, fun, motivation and suggestion for improvement. Moreover, they answered a comprehension question first without AR and then with the help of the AR experience. The question "What is the direction of the axial force on the second gear wheel of the helical gear?" has been answered in a peer-review setting to check their learning success.

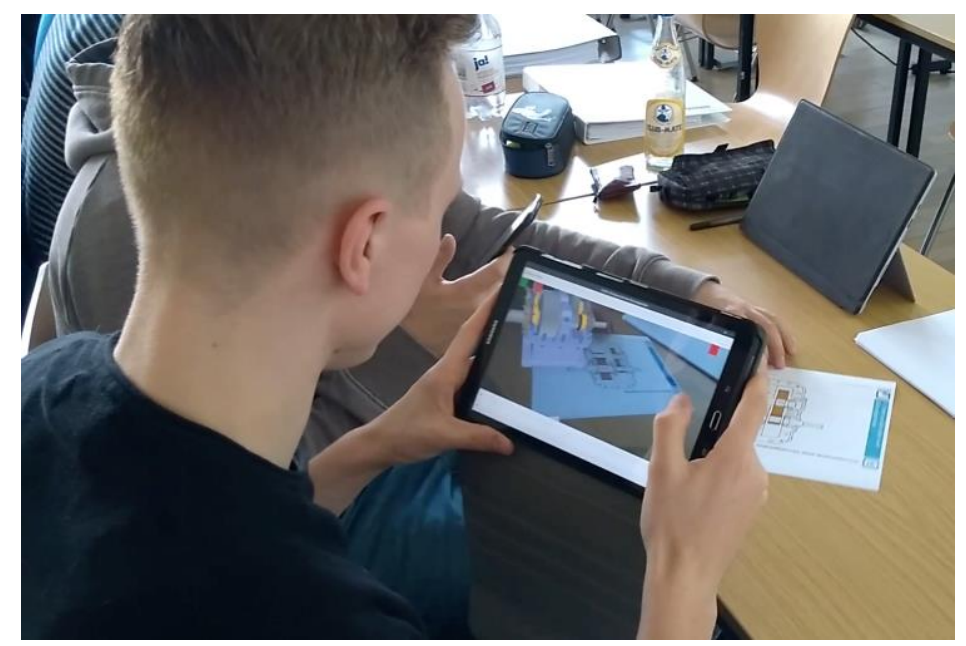

Figure 1. Application of an AR experience which includes a two-stage gear unit during a "machine elements" lecture

The experiment revealed the following advantages and disadvantages:

The handling in the AR experience with the used software has been evaluated as good and simple.

The AR experience increased the motivation of the students and the teaching content was available at flexible times. In addition, the AR experiences supported self-learning, as many students could imagine using the AR experiences for preparing and following up on the course material. Not good were the extra costs, the high information density in the AR experience and the invisible learning success for the above mentioned test question compared to conventional teaching.

There are multiple reasons for the students giving the wrong answer to the test question. On the one hand, the period of time that the students had for understanding the content of the AR experience in order to answer the test question afterwards was too short. On the other hand, the information density was too high, so that the application was not completely meaningful in this case. Hence no significant success of learning could be determined. Due to these difficulties in creating AR experiences that are conducive to learning, further research has been done in the field of pedagogy.

As a result of this research, a flowchart has been developed in this paper that allows (AR inexperienced) tutors to create AR experiences that fit the learning objective. With the help of this flowchart, it is intended to achieve that basic lectures can be enriched with AR in a targeted manner and thus be made more attractive in order to promote more efficient learning. However, this paper still represents a feasibility study that is meant to lay the foundation for sophisticated studies, since the effect of the flow chart developed here could not yet be scientifically verified in practice.

\section{Requirements for mobile augmented reality applications}

The following eight items needed to be concerned before creating an augmented reality experience for the application in higher education.

1. Hardware:

With appropriate hardware, the real environment can be captured by camera and shown on a display. Virtual information can then be overlaid on it. Examples for appropriate hardware are: Smart Glasses (e.g. "Google Glass"), Augmented Reality Glasses (e.g. "Microsoft HoloLens") and 
smartphones or tablets (Thomas et al., 2018). Smart Glasses have a deeper immersion (i.e. a state of consciousness of feeling inside the virtual environment) than smartphones or tablets, but ARGlasses have the deepest (Tscherner, 2018; Weber and Herrmann, 2008). In this work, smartphones and tablets have been chosen as the most suitable hardware. The reason for this is that there is no need for a deeper immersion into the virtual information in classes since the real surroundings are still to be registered by the students. Moreover, nearly every student of the observed university already owns a tablet or smartphone and uses it regularly during lectures. Nowadays, smartphones even have a suitable image quality (Tscherner, 2018).

2. Software:

First, the software is used to build the AR experience(s). Afterwards the app on the smartphone loads the AR experience data from the cloud. For this project, "Vuforia Studio" has been chosen as programming software while "Vuforia View" has been chosen as an app for the user (advantages have been shown in the introduction). Both software is from PTC, which is a big global player for CAD and AR in the world. PTC creo is already used for CAD instructions at Ostfalia University, so students know how to use it.

3. Expenditures:

As the choice of hardware does not generate any extra costs (Lai, 2017), only the license for the "Vuforia Studio" software and the creation of the augmented reality experience itself (possible labour costs) need to be considered. The approximate duration to assemble a whole AR experience including a CAD model with medium to large complexity is shown in Table 1. It shows the needed working hours to design a complete AR-experience as reference. The reference hours have been recorded during the design of the showcase "profil displacements within a two-stage gear unit" as shown in Figure 1. The task has been performed by a student worker. The basis was an 2D sectional view of the dedicated gear. The real effort varies based on the complexity and size of the concrete AR-experience. Because, if more standard parts are used CAD modelling hours would be less, and if complex (freeform) surfaces are needed CAD hours would be more.

Table 1. Approximate duration to assemble a whole AR experience

\begin{tabular}{|l|l|}
\hline Performed task & Direct labour \\
\hline a) Concept creation & 24 hours \\
\hline b) CAD modelling & 80 hours \\
\hline c) Layout of the worksheet & 8 hours \\
\hline d) AR programming & 16 hours \\
\hline e) Validation and optimizing & 8 hours \\
\hline Altogether attended time of one AR experience & $\mathbf{1 3 6}$ hours \\
\hline
\end{tabular}

\section{Operating mode:}

The used operating mode is a marker-based recognition (Tscherner, 2018). In order to make virtual content visible on smartphones or tablets, the real environment of the user or a point of reference must be recognized by the device. Thus, the surrounding room must be lightened adequately and changes of light, shadows, reflections, etc. must be avoided (Tscherner, 2018). Furthermore, the device must be connected to a WIFI source if the AR-experience is not downloaded yet (Syazani, 2014). As soon as these requirements are satisfied, image points of the real objects are recognized automatically by the camera and are then overlaid with virtual information (Tscherner, 2018). Examples for such image points are "QR-codes". The coordinate origin of this "QR-code" is the reference for all virtual contents, which can appear in different distances from it (Tscherner, 2018).

\section{How the brain works:}

To ensure an efficient study and a meaningful application of augmented reality experiences, it is important to know how humans learn. New educational information can only reach the long- term memory if it has been registered and successfully processed in the working memory before. This bottleneck limits the further processing of information and thus the learning 
velocity. For this reason, a long-term "deliberate practice" is inevitable. Moreover, the "dualcoding-theory" claims that combining written and spoken language helps the brain to gain factual knowledge and practical qualifications better. The long-term memory establishes a linguistic and a visual representation and links them. Thereby the knowledge network becomes more extensive, dense and stable. It gets easier for the brain to recall the gained information, which improves the quality of education. (Schneider and Mustafic, 2015)

6. Factors of influence in university teaching

The graph in Figure 2 shows the relationship between the educational objectives, the students and lecturing tutors in the teaching-learning process. At first, special educational objectives must be derived from the content of teaching (didactics). The learning content is then conveyed by appropriate methodology (e.g. AR as learning media) to achieve the educational objectives. (Luttermann, 2000)

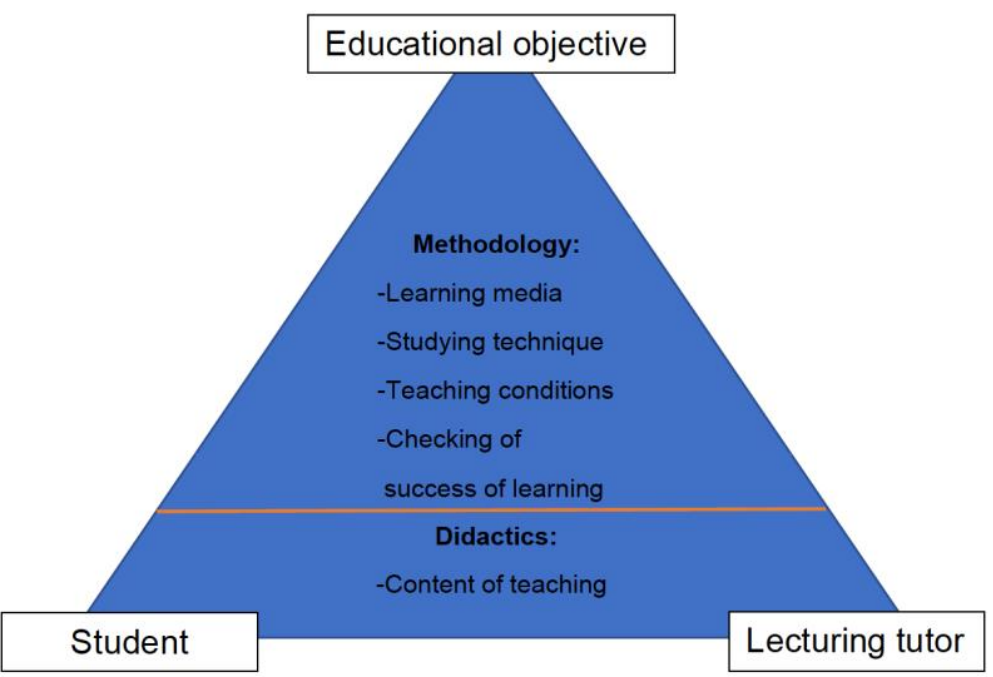

Figure 2. Factors of influence in university teaching based on (Luttermann, 2000)

7. Classification of educational objective:

According to Bloom, educational objectives can be classified into different layers depending on their degree of complexity (1-6). In order to distinguish between the different layers, every cognitive result of learning can be linked with a certain behaviour (table below). Each teaching content can then be related to a certain layer (cp. Table 2).

Table 2. Learning target taxonomy according to Bloom (Rosza, 2012)

\begin{tabular}{|c|c|c|}
\hline $\begin{array}{l}\text { Degree of } \\
\text { complexity }\end{array}$ & $\begin{array}{l}\text { Cognitive result of } \\
\text { learning }\end{array}$ & Behaviour component \\
\hline 6 & Evaluation & $\begin{array}{l}\text { Established opinions can be made: } \\
\text { (to) judge, weigh, decide, discuss, comment, review, classify, etc. }\end{array}$ \\
\hline 5 & Synthesis & $\begin{array}{l}\text { Elements can be compounded new again: } \\
\text { (to) conceive, plan, integrate, reason, prove, design, etc. }\end{array}$ \\
\hline 4 & Analysis & $\begin{array}{l}\text { Facts can be parsed: } \\
\text { (to) derive, find out, subdivide, discover, interpret, check, etc. }\end{array}$ \\
\hline 3 & Practice & $\begin{array}{l}\text { Rules and principles can be applied: } \\
\text { (to) use, edit, detect, constitute, engineer, draw, calculate, etc. }\end{array}$ \\
\hline 2 & Understanding & $\begin{array}{l}\text { New information can be processed and pigeonholed: } \\
\text { (to) determine, apply, describe, identify, compare, assign, etc. }\end{array}$ \\
\hline 1 & Knowledge & $\begin{array}{l}\text { Contents can be remembered: } \\
\text { (to) name, define, reproduce, report, list, sketch, answer, etc. }\end{array}$ \\
\hline
\end{tabular}


The degree of complexity builds up hierarchically. However, the brain does not necessarily operates in one layer at a time or needs to edit these layers in the given order (1-6) to learn new content. For example, evaluation does not necessarily have to be the last step in thinking or problem-solving. In contrast, it precedes the acquisition of new knowledge. However, evaluation forms a major link to the affective area (joy, fun). (Bloom et al., 1984) Consequently, the same learning medium might support different layers or different media support one layer. It depends on the learning context and objective.

8. Types of intermediations of educational objectives

Conventionally, educational objectives are taught with the help of 2D-elements (e.g. lecture notes, pictures), animations (e.g. video sequences), real exhibits or communication and interaction (e.g. teamwork practices, simulation games, short presentations of students). With AR experiences, educational objectives can be taught in a similar way. Table 3 shows in its first row the currently used learning media in the lecture "machine elements". Generally speaking, different media are used in different complexity levels. As similarly explained in item 7, media from the lower levels can also be part of a more complex learning setting. The second row shows how AR can support the students for the same complexity level. As an example, real exhibits, which cannot be procured, can be replaced adequately by virtual 3Dobjects and used for analysis. In addition, the application of AR experiences makes an interaction of virtual and real information possible, which makes simulations more realistic and lets the user experience the content hands-on.

Table 3. Support of conventional media by AR experiences

\begin{tabular}{|c|c|c|c|c|c|c|}
\hline & \multicolumn{6}{|c|}{ Complexity of cognitive learning results } \\
\hline & Knowledge & Understanding & Practice & Analysis & Synthesis & Evaluation \\
\hline 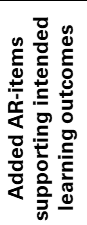 & \begin{tabular}{|c|} 
interactively adding \\
different context \\
specific annotations on \\
the same 2D pictures
\end{tabular} & \begin{tabular}{|} 
interactively managing \\
$3 \mathrm{D}$ animation \\
sequences, explosion \\
views and highlights
\end{tabular} & $\begin{array}{l}\text { stepwise highlighting } \\
\text { the next formulaand } \\
\text { interdependencies in } \\
\text { the formulary }\end{array}$ & $\begin{array}{c}\text { discovering the } \\
\text { functionality of } 3 \mathrm{D} \\
\text { models and tangibly } \\
\text { check the influence of } \\
\text { modifications and } \\
\text { variations }\end{array}$ & $\begin{array}{c}\text { adding different virtual } \\
\text { 3D solution elements to } \\
\text { real elements and } \\
\text { checking for collision or } \\
\text { examine the assembly } \\
\text { process }\end{array}$ & $\begin{array}{c}\text { reviewing a predefined } \\
\text { solution by interactively } \\
\text { overlying process } \\
\text { information for different } \\
\text { use cases }\end{array}$ \\
\hline
\end{tabular}

\section{Pedagogical findings to accomplish an efficient learning}

This section shows important and proven positive factors of influence in higher education and furthermore explains how they can be achieved by AR. Figure 3 structures and summarises the findings from the subsequent references. The main objective of AR, an "efficient learning", is satisfied by the implementation of the targets "preparation from the academics", "deliberate practice" and "self-efficacy conviction" with appropriate methodology. Each methodology can be achieved or improved by means of AR experiences. For example, motivation can be increased through AR experiences, as it is a new learning method with a playful character. This has been confirmed by initial surveys (cp. introduction). Time sovereignty is possible due to the temporal flexibility of the AR experiences in the download offline mode without internet. And feedback on questions can also for example provided in high quality with the help of AR experiences by means of visual and realistic explanations. From left to right the focus shifts from the lecturing tutor to student perspective. In the field of "deliberate practice", there is a transitional area which should make it clear that both, students and lecturing tutors can contribute to this area.

The first important factor of influence is the preparation of a course by the lecturing tutor. In addition to the clarity and understandability of the learning content in the course, this factor of 
influence also includes a specific definition of the educational objectives (classification see Table 2). Achieving the educational objectives should also be verifiable. A clear goal naming and explanation of how to achieve them independently will stimulate successful learning. (Schneider and Mustafic, 2015) The second important factor of influence is "deliberate practice", which means an intelligent practice and concerns lecturing tutors and students. To practice intelligently means not only to practice a lot (quantitatively), but also to identify and work on one's own weaknesses continuously and purposefully (Schneider and Mustafic, 2015). These weaknesses must be detected in a feedback by a second person, i.e. the lecturing tutor or fellow students. Subsequently, new and slightly more demanding educational objectives should be set again and again (Schneider and Mustafic, 2015).

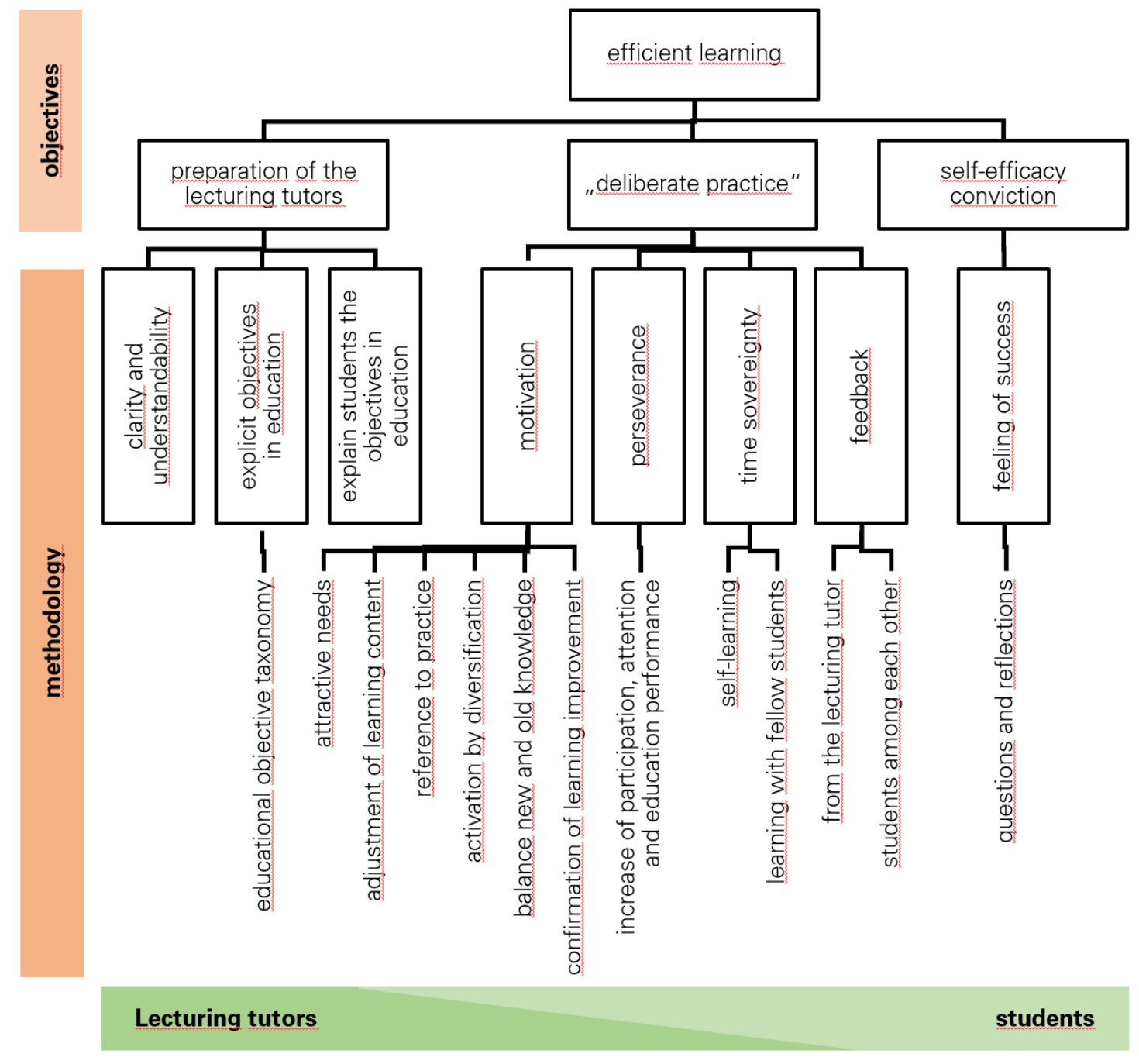

Figure 3. Objectives of AR for an efficient study

Precondition for deliberate practice are time, perseverance, and motivation (Schneider and Mustafic, 2015). Motivation influences the intensity of learning, activity of students, and participation in educational courses (Rosza, 2012). The motivation can be improved by the following items:

- The more cognitive and social emotional needs (like fun, pleasure, etc.) are activated (Rosza, 2012). AR experiences are fun, since they allow direct interaction.

- The more the content is appropriate for the level of aspiration and learning conditions (Rosza, 2012). AR experiences can be used for any length of time to satisfy individual learning requirements by time sovereignty. 
- The clearer the practical relevance and usability of the learned content are (Rosza, 2012). Scenarios can be modelled and implemented in AR experiences more realistically by combining virtual and real information.

- The more learning difficulties are discussed and dismantled (Rosza, 2012). Purposefully used AR experiences can clarify difficulties individually.

- The better the balance between known and new learning content is (Rosza, 2012). AR experiences provide a known content in the real environment and new, changing and advancing content as a virtual "add-on".

- The more learning progress is recognized and confirmed (Rosza, 2012). This can be done in particular by the correct answering to quick voting surveys about AR experiences.

The third and last very important factor of influence on good higher education is the self-efficacy conviction of students (Schneider and Mustafic, 2015). The self-efficacy conviction indicates to what extent persons (for example students) are convinced that they can master new and difficult situations due to their own competences (Schöber et al., 2018). Therefore, the self-efficacy conviction is an inner attitude based on self-assessment. This can be strengthened by recurrent feelings of success for example by solving questions or exercises correctly. Thereby, open questions that require independent reasoning, analysis, assessment, or design are much more effective than closed questions, if they are only intended to verify factual knowledge (Schneider and Mustafic, 2015).

\section{Application of the flowchart to augmented reality experiences}

The purpose of the flowchart is to provide a flexible guide to the creation of meaningful AR experiences in order to achieve an efficient learning. The whole flowchart is subclassified in two parts. Due to limited space, this contribution will just give an overview on the flowchart with a focus on the first part. The complete flowchart can be requested at the authors. Every part of the flowchart contains questionnaires that guide the user to a recommendation on the best way of intermediation of the desired teaching content.

The first part of the flowchart (Figure 4) deals with the question of whether it makes sense to create an AR experience with an additional amount of time (cp. Table 1), or whether teaching contents should be better communicated conventionally. At first, educational objectives must be formulated specifically and concretely. Only then the learning target level can be determined (Table 3) and certain prerequisites for the desired level can be considered (an example can be found in section 5). Afterwards, the first questionnaire (red box) is handled. The short headings in Figure 4 (1.1, 2.1, etc.) represent a set of concrete questions. The background of all questions is to check whether the advantages of AR compared to conventional learning methods can be applied in a certain case. If all questions are answered "no", the teaching content should clearly be presented conventionally.

For example, the corresponding questions 1.1 and 1.2 are:

- 1.1 (Realizable procurement): Are real exhibits not or only difficult to procure?

- 1.2 (Time sovereignty): Is it important that every student has an exhibit available while the lecturing tutor explains relevant information? OR: Is it important that each student can simultaneously solve a problem by using an exhibit?

In this case, the advantage of AR compared to exhibits is considered. Answering the first question 1.1 with "yes" alone is not yet a prerequisite for the creation of an AR experience, because the teaching content can also be conveyed without an exhibit or does not justify additional expenditures. Therefore the second questionnaire has been created (yellow box), which serves to indicate where to set focus on in the AR experiences and deepens the questions from the first questionnaire. Thus, the numbering is corresponding (e.g. 1.1 is deepened in 1.2). In this case, it only makes sense to create an AR experience when the second question 1.2 has also been answered with "yes". In the concrete observed lecture exhibits are limited in their number, thus not available to all of the students at the same time. However, for better educational intermediation, they should be available during the instructor's explanation. 
All remaining questions of the flowchart are listed on a form, which is available on request at the authors as well. The more questions are answered with "yes" the higher the probability that educational objectives shall be communicated with AR experiences.

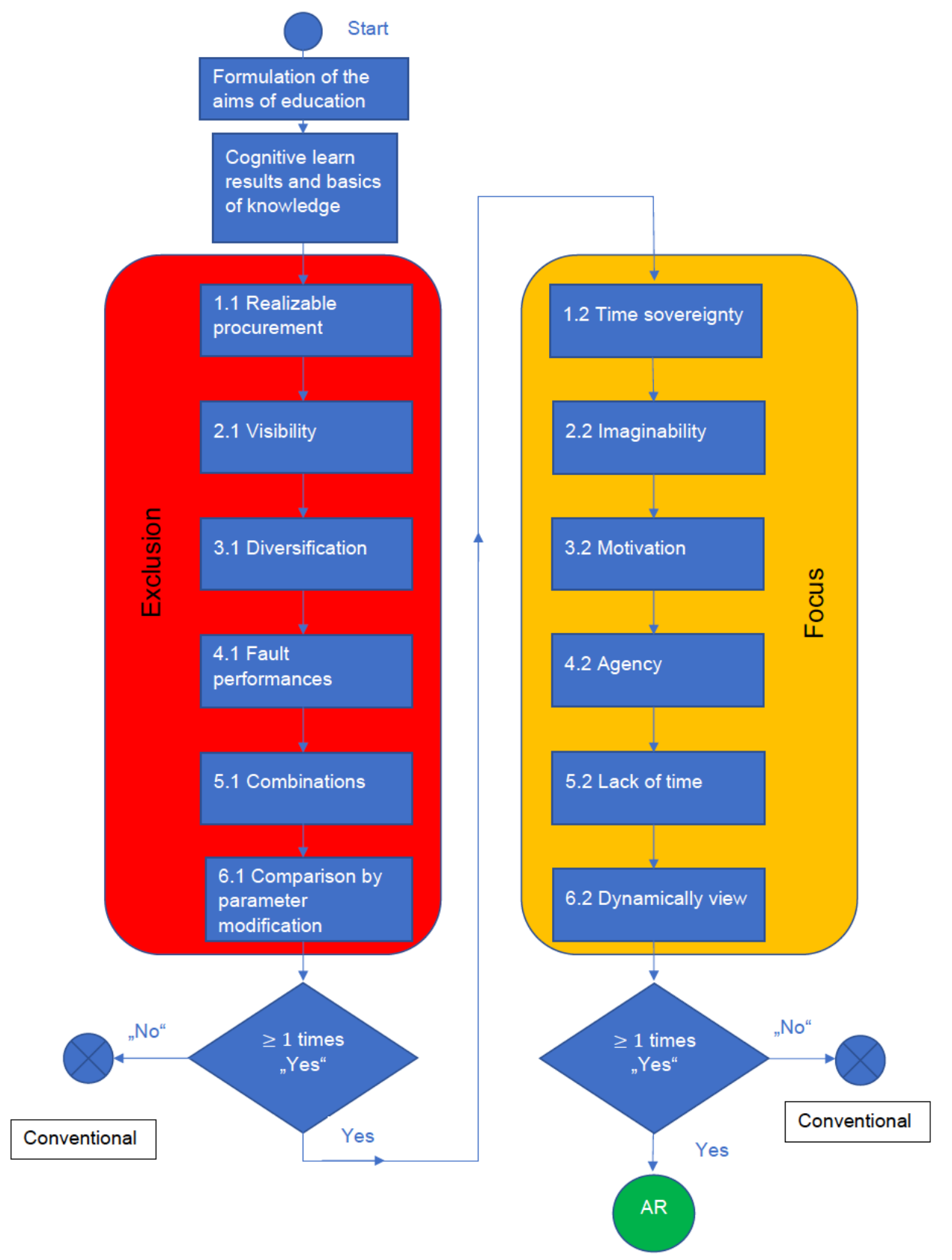

Figure 4. General flowchart for development of AR experiences in higher education part 1

The second part of the flowchart (not illustrated here) deals with the way teaching contents in AR experiences should be conveyed. It is made up of four sets of specific questions of which one set is for 2D AR experiences, one for 3D AR experiences, one for interactive AR experiences (real elements and virtual elements interact) and one for animation in AR experiences. If a certain questionnaire, e.g. the one for 2D AR experiences is answered at least one time with "yes", the considered kind of AR experience (here 2D AR) should be applied. If several questionnaires are answered with at least one "yes", it makes sense to use a hybrid type in the AR experiences to be created. When creating the AR experiences, it is essential to ensure that the density of information is kept as low as possible. Too much information on the small mobile screen will overcharge the user. In order to achieve this, 2D information is "outsourced" to the required paper-worksheet, which also includes the "QR-code" (Figure 5). 


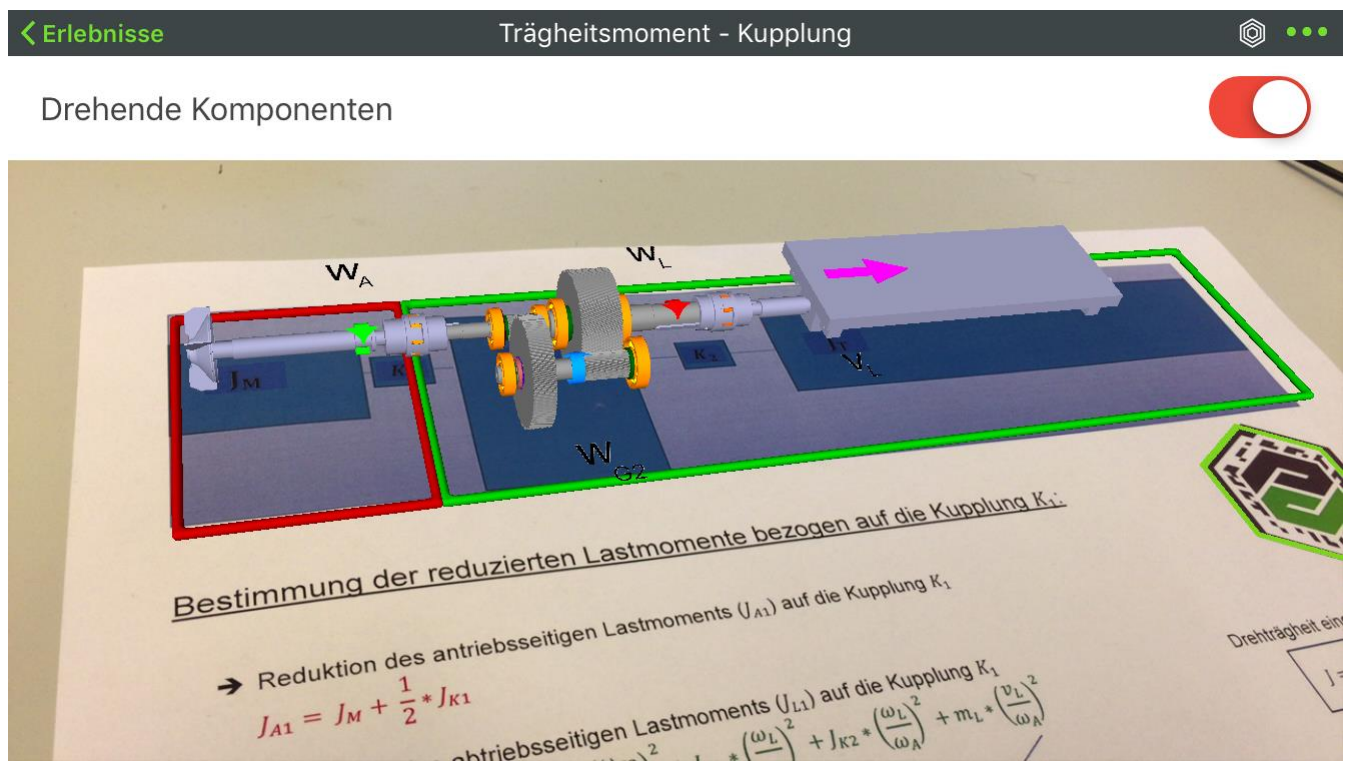

Figure 5. AR experience "Load torques apropos of different clutches"

\section{Conclusion and general validity of the flowchart}

Up to now, three AR experiences have been generated using the flowchart, which has shown that the flowchart is coherent for the use of different topics in the field of machine elements, i.e. gears, bearings and clutches. This section presents one of these AR experiences, that was created following the instructions of the flowchart in order to check its function. However, a sophisticated study has to be done in order to validate, if the created AR experience leads to a significant learning success of the students that will be testing it. For this study, the learning results of 40 students using conventional learning methods has already been recorded and as a next step the learning success of the equal amount of students using the AR experience has to be investigated. Then the two effects have to be compared.

According to the first step of the flowchart, the intended learning outcome has been determined as "The students are able to reduce a complex system with different rotating and linearly moving masses of different moments of inertia into a two-mass oscillator. With this knowledge the drive side and the load side reduced moments of inertia, referring a specific clutch, are to be calculated and then the students should decide between different given calculating results for the correct one." Through this concrete formulation of the intended learning outcome, it is assigned to the degree of complexity of "evaluation" in step two of the flowchart. The following basics result from the degree of complexity of the intended learning outcome and are necessary for its achievement:

a) Knowledge about the structure and function of a clutch (Table 2, no.1).

b) Physical understanding of the inertia of masses and Newton's law (Table 2, no.2).

c) Analytical capability of a technical system with power unit, clutch, gear box, clutch, and output (Table 2, no.4)

Afterwards, in the third step of the flowchart the first questionnaire has been edited, which lead to the following findings. It is important to make hidden information visible, such as the moved inertias and their velocities (answer to 2.1). Furthermore, the experience of the lecturing tutor has shown, that the calculation of the reduced moment of inertia is always a difficult topic for the students and as a consequence, the dimensions of clutches are often designed inadequately (4.1). Moreover, it is needed to switch between different clutches of the system (left and right of the gear box) to calculate the different reduced moments of inertia (6.1). By answering these three questions with "yes", the second questionnaire had to be edited. This has shown, that it is important that every student can decide for the correct calculating result individually by working with an own model of the system (1.2). In addition, the external motivation should be enhanced, since this topic is introduced at the very end of the semester (3.2). Conventional teaching methods have been evaluated as less illustrative, which makes a theoretical abstraction more difficult for the students (4.2). Due to these given "yes", it is meaningful to create an 
AR experience with focus on the connection between the real components and their theoretical abstraction so that the students can reduce the information to a substitutional system. In addition, the application of the second part of the flowchart suggested to outsource the theoretical abstraction (moments of inertia) from the AR experience to the worksheet in order to minimize information density in the AR experience. The virtual 3D models in the experience are corresponding to their theoretical abstraction on the worksheet. With the slide on the top right it is possible to switch the view between the physical display of the components and the display of their moving parts (last view is shown in Figure 5). All up to now created AR experiences are from the area of the "machine elements" lecture. Therefore, this area is considered as a reference for the generated questions of the flowchart. Nevertheless, a general formulation of the questions was emphasized, so that the topic for the creation of AR experiences could also come from another area. However, this still needs to be further examined. It is planned to also create AR experiences for "design methodology" and "structural analysis", so that it can be checked whether the flowchart is suitable for different engineering disciplines and different lecturers. The practical experience from applying the flowchart in different disciplines will help to improve it. Thereby, more questions might be developed that can then be added into the existing questionnaires, which keeps the flowchart valid throughout its application.

Overall, the flowchart seems to be a good and flexible prerequisite for achieving an efficient study with $\mathrm{AR}$, because a concrete naming and classification of the educational objectives provides an indication of suitable intermediation of them.

\section{References}

Bloom, B. et al. (1984), Taxonomy od educational objectives: the classification of educational goals, Longman. Campbell, M. et al. (2019), White Paper: The State of Industrial Augmented Reality 2019, PTC, PTC.com.

Davies, P. (2018), The Boeing Augmented Reality Kit (BARK) in Airplane Manufacturing. [online], AWE-Augmented World Expo. Available at: https://www.youtube.com/watch?v=jItpoNiOMWs (accessed 27.10.2019)

Lai, Y.-C. (2017), "Is Augmented Reality a new paradigm in design education when educational subsidy decreases", International conference on engineering and product design education 7\&8 september 2017, Oslo and Akershus University College of Applied Sciences, Norway.

Luttermann, C. (2000), Multimedia in der betrieblichen Weiterbildung, Integration neuer Technologien, Diplomica, Norderstedt.

Probst, A. and Ebner, M. (2018), "Introducing Augmented Reality at Secondary Colleges of Engineering", International conference on engineering and product design education 6\&7 september 2018, Dyson School of Design Engineering, Imperial College, London, United Kingdome.

Rozsa, J. (2012), Aktivierende Methoden für den Hochschulalltag, Heidelberger Hochschulverlag, Heidelberg.

Schneider, M. and Mustafic, M. (2015), Gute Hochschullehre: Eine evidenzbasierte Orientierungshilfe, Springer, Berlin. https://doi.org/10.1007/978-3-662-45062-8

Schöber, C. et al. (2018), "Reciprocal effects between self-efficacy and achievement in mathematics and reading", Learning and individual differences, Vol. 63, pp. 1-11.

Slater, M. (2017), "Implicit Learning Through Embodiment in Immersive Virtual reality”, In: Liu, D., Dede, C., Huang, R. and Richards, J. (Eds.), Virtual, Augmented, and Mixed Realities in Education, Springer, Singapore, pp. 23. https://doi.org/10.1007/978-981-10-5490-7

Syazani, S., Junaidi, A. and Chyi, K.D. (2014), "A Resource-Independent Marker-Based Augmented Reality Application", In: Das, V.V. and Elkafrawy, P. (Eds.), Signal Processing and Information Technology, Springer, Heidelberg, New York, Dordrecht, London, pp. 204. https://doi.org/10.1007/978-3-319-11629-7

Thomas, O., Metzger, D. and Niegemann, H. (2018), Digitalisierung in der Aus- und Weiterbildung, Springer Gabler, Berlin. https://doi.org/10.1007/978-3-662-56551-3

Tscherner, M. (2018), Visualisierung von Augmented-Reality-Inhalten [Bachelor Thesis], Universität Berlin.

Weber, M. and Herrmann, M. (2008), “Advanced Hands and Eyes Interaction”, In: Mühlhäuser, M. and Gurevych, I. (Eds.), Handbook of Research on Ubiquitous Computing Technology for Real Time Enterprises, IGI Global, Hershey, pp. 454. https://doi.org/10.4018/978-1-59904-832-1.ch019 